\title{
Erratum
}

\section{A Highly Sustainable and Active Catalyst for Suzuki-Miyaura Reaction: Palladium-Supported Ionic Liquid Catalyst (SILC) Coated with Polymer}

Hisahiro Hagiwara,* Kota Sato, Takashi Hoshi, Toshio Suzuki Synlett 2011, 2545.

On page 2546, left column, line 6 from the bottom, PET-Pd-SILC (4A) should be PS-Pd-SILC (5A). Further, on page 2546, right column, Figure 3, caption of the left picture should be PS-Pd-SILC (5A). The caption of Figure 3 should be "SEM images of cross sections of PS-Pd-SILC (5A) and PET-Pd-SILC (4S) (20 kv, x1,000)". 